\title{
Éditorial: Énoncé de politique sur les défis de la prestation des services de santé mentale aux personnes âgées
}

\section{Introduction}

Les personnes âgées atteintes de troubles mentaux sont une population particulièrement vulnérable qui a des besoins uniques en matière de soins. La restructuration actuelle des systèmes de santé vise l'affectation et l'utilisation efficiente et efficace des ressources. La collecte des données, l'évaluation des programmes et l'assurance de la qualité sont essentielles à la réforme des services de santé. Alors que s'opère cette réorganisation, il est important de tenir compte des besoins spéciaux des populations vulnérables et d'articuler clairement les principes sur lesquels reposent leurs soins.

Nous souhaitons présenter ici un aperçu des besoins des personnes âgées atteintes de troubles mentaux et proposer certaines stratégies éprouvées pour répondre à ces besoins et adopter des politiques et programmes rationnels permettant de dispenser des soins efficaces et compatissants. Nous examinerons les défis que présente la prestation de services de santé mentale aux personnes âgées. Toutefois, les limites du présent document ne nous permettent pas de présenter un examen approfondi de tous les programmes dispensant des services de ce type au Canada. Nous souhaitons pourtant encourager un changement dans les pratiques et faire mieux comprendre les principes régissant la prestation de soins appropriés aux personnes âgées atteintes de troubles mentaux. À l'heure actuelle, nombre des travaux de recherche portant sur la santé mentale des aînés ${ }^{1}$ ne sont pas vraiment appliqués dans la pratique. Par ailleurs, ce que les personnes atteintes de troubles mentaux et leurs familles pensent des services qu'elles reçoivent peut s'avérer très instructif et faciliter la prestation de services accessibles et adaptés. L'information, la sensibilisation et la formation sont essentielles si l'on veut dispenser des services appropriés.

Nous commencerons par donner un bref historique du problème et des principes de soins, puis nous discuterons de la nécessité d'avoir des services de santé mentale s'adressant spécifiquement aux besoins des personnes âgées. Nous expliquons pourquoi nous sommes convaincues que le modèle de soins le plus approprié pour les aînés atteints de troubles mentaux est un modèle de soins social, qui reconnaît l'importance des facteurs psychologiques, sociaux, physiques et environnementaux sur la santé mentale. Dans le chapitre qui suit, nous fournirons un aperçu des principes régissant la prestation de soins appropriés aux personnes âgées atteintes de troubles mentaux, puis nous verrons comment appliquer ces principes directeurs aux services de santé mentale destinés à cette clientèle.

\section{Aperçu historique}

Au Canada, près de 12 pour cent de la population est âgée de 65 ans ou plus. Selon l'Association médicale canadienne (1987) quelque 30 pour cent d'aînés canadiens ont besoin, à un moment ou à un autre, de services de santé mentale. De même, Santé et Bien-être social Canada (1991) cite un taux de prévalence de 25 pour cent. Gurland et Cross (1982) suggèrent que 15 à 20 pour cent des personnes âgées pourraient avoir besoin des services psychiatriques. Blazer et Maddox (1982) indiquent que 5 à 10 pour cent des aînés vivant dans la communauté souffrent de troubles psychiatriques graves et entre 10 et 40 pour cent de troubles mentaux légers à modérés.

Au début des années 1980, la population des personnes âgées suscite un regain d'intérêt de la part des gouvernements fédéral et provinciaux, des établissements de santé, des organismes communautaires et des universités, comme en témoigne le nombre croissant de groupes d'intérêt, conférences, subventions, publications et groupes de travail spécialisés qui se forment (Béland, 1997). Il n'est donc pas étonnant que Santé et Bien-être social Canada traite des questions de santé mentale des aînés dans plusieurs de ses publications, telles que Troubles mentaux chez les personnes âgées au Canada. Considérations démographiques et épidémiologiques (1991); Guide pour l'établissement de normes régissant les services dispensés aux personnes âgées atteintes de troubles mentaux dans les établissements de soins prolongés (1990); Lignes directrices pour la prestation de services complets aux personnes âgées atteintes de troubles mentaux (1988a); et La santé mentale des Canadiens: vers un juste équilibre (1988b). Ces initiatives fédérales ont une influence considérable sur l'élaboration de lignes directrices et de cadres de planification au niveau provincial. Dans de nombreux territoires de compétence, les questions de santé (y compris les services de santé mentale) relèvent désormais des bureaux régionaux de la santé. On pense, en effet, qu'en ayant un système administratif unique, tel qu'un office régional de la santé, on résoudra cer- 
taines des difficultés posées par les systèmes de santé complexes et polyvalents (Shulman, 1991). Toutefois, pour y parvenir, il faut que ces nouvelles entités se familiarisent avec les modèles actuels de prestation des services de santé mentale, les principes sur lesquels ils reposent et les limites de leur application pratique. L'Organisation mondiale de la santé et l'Association mondiale de psychiatrie ont récemment publié ensemble un énoncé de consensus technique sur l'organisation des soins destinés aux personnes âgées atteintes de troubles mentaux. L'énoncé offre des conseils à ceux qui élaborent des politiques et programmes dans ce domaine (Organisation mondiale de la santé et Association mondiale de psychiatrie, 1998). La British Columbia Psychogeriatric Association, une organisation multidisciplinaire formée de professionnels oeuvrant dans le secteur de la psychogériatrie, a énoncé une série de principes de soins en psychogériatrie (British Columbia Psychogeriatric Association, 1999). Ces principes de soins aux aînés atteints de troubles mentaux et à leurs familles servent de guide pour la planification et l'élaboration de services de santé destinés spécifiquement à cette clientèle. Les services doivent être axés sur les objectifs et les clients, être souples, complets, responsables et adaptés aux besoins des personnes âgées.

\section{Services de santé mentale destinés spécifiquement aux personnes âgées}

La santé mentale, telle que la voit les consommateurs, les familles et les spécialistes et telle qu'elle est définie dans La santé mentale des Canadiens: vers un juste équilibre (Santé et Bien-être social Canada, 1988b), est:

la capacité de la personne, du groupe et du milieu d'établir des liens qui favorisent le bienêtre, le développement et l'utilisation optima des capacités mentales (cognitives, affectives et relationnelles), l'atteinte d'objectifs personnels et collectifs dans le respect de la justice, et l'atteinte et la préservation des conditions d'égalité fondamentale (p.7). (Traduction libre)

Les problèmes de santé mentale résultent de la perturbation des relations entre la personne, le groupe et le milieu et peuvent venir de la personne (par ex., maladie physique ou mentale, mécanismes d'adaptation insuffisants), ou de causes extérieures (par ex., conditions environnementales, structures sociales ou dynamique des relations) (Santé et Bien-être social Canada, 1988b). Voici une définition plus précise des troubles mentaux «une maladie qu'il est possible de diagnostiquer médicalement et qui entraîne une grave déficience des aptitudes cognitives, affectives ou relationnelles de la personne» (Traduction libre) (Santé et Bien-être social Canada, 1988b, p.8). Cependant, même lorsqu'elle se traduit par de graves troubles psychiatriques, cette déficience n'est pas le seul déterminant de la santé mentale. Les troubles psychiatriques coexistent avec des problèmes de santé mentale qui doivent aussi être réglés pour atteindre un niveau de santé mentale optimal (Santé et Bienêtre social Canada, 1988b).

Le besoin en services de santé mentale s'adressant spécifiquement aux personnes âgées est bien établi dans la documentation spécialisée et vise trois grands secteurs: 1) les différences liées à l'âge; 2) les obstacles à l'accès aux soins; 3 ) les facteurs de développement et les facteurs sociaux liés à l'âge. Jusqu'à tout récemment, les problèmes de santé mentale des personnes âgées n'étaient pas considérés comme différents de ceux des autres adultes.

\section{Différences liées à l'âge}

La dépression, la démence, les troubles délirants et le délire sont les formes les plus courantes de troubles mentaux présentés par les personnes âgées. Bien qu'ils puissent être rencontrés chez les adultes plus jeunes, ils sont liés à l'âge. La démence et le délire, par exemple, sont beaucoup plus fréquents chez les personnes âgées que chez les autres adultes. La dépression et les troubles délirants peuvent se manifester de façon très différente chez les aînés que chez les adultes plus jeunes.

La démence est liée à l'âge. Elle touche moins de 1 pour cent de personnes de moins de 65 ans, mais 2,5 pour cent de personnes âgées de 65 à 74 ans, 11 pour cent de sujets entre 75 et 84 ans et 35 pour cent de personnes de plus de 85 ans (Groupe d'étude sur la santé et le vieillissement au Canada, 1994). Elle fait donc partie des problèmes que les professionnels de la santé, les fournisseurs de services et les décideurs doivent comprendre. Les personnes atteintes de démence ont besoin de divers niveaux et types de soins, selon la gravité de leur trouble et les symptômes qu'elles présentent (par ex., caractéristiques affectives ou psychotiques, problèmes de comportement). Soigner ces malades est un grand défi pour les familles et le personnel spécialisé. Les fournisseurs de soins ont besoin de beaucoup d'aide et de soutien (par ex., sur le plan de la formation et de la santé physique et mentale). La complexité des symptômes de démence et leur incidence sur la société nécessitent que les professionnels de la santé aient de vastes connaissances et compétences pour évaluer l'éventail compliqué des facteurs biologiques, psychologiques, sociaux et environnementaux qui leur permettent de dispenser des soins optimaux et de gérer efficacement les problèmes en toute circonstance.

On a décrit le délire (c.-à-d., les variations de conscience, d'attention et d'orientation) comme le trouble psychique mortel, mais potentiellement réversible, le 
plus courant chez les personnes âgées (Beresin, 1988). Bien que le délire soit considéré comme un urgence médicale, il est rarement traité comme tel, même en milieu de soins actifs. De nombreux troubles de santé qui seraient relativement bénins chez des personnes plus jeunes et en meilleure santé (par ex., infections de tout genre, rétention urinaire, constipation, fécalome) peuvent provoquer le délire chez les personnes âgées. Voici quelques-uns des facteurs déclenchants du délire: déficience cognitive préexistante; changements, liés à l'âge, dans la pharmacocinétique et la pharmacodynamique des médicaments; résistance moindre aux maladies aiguës; gravité des maladies chroniques; troubles du sommeil; isolement sensoriel; hypertension sensorielle; déficience visuelle et auditive; stress psychosocial; deuil; ou déracinement dans un milieu étranger (Lipowski, 1989). Comme elles prennent davantage de médicaments d'ordonnance que les jeunes, les personnes âgées courent un risque plus grand d'être victimes d'une interaction médicamenteuse grave et d'avoir des réactions indésirables à certains médicaments pouvant causer le délire (Lee, 1996). Le dépistage et la prévention précoces du délire nécessitent une connaissance spécialisée des facteurs prédisposants et de leur gestion si l'on veut éviter les hospitalisations inutiles et/ou les décès. Il est donc essentiel que les fournisseurs de services de santé aient la formation nécessaire pour reconnaître et gérer le délire.

De nombreux facteurs liés spécifiquement à l'âge peuvent compliquer le diagnostic de la dépression chez les personnes âgées. En effet, les symptômes de tristesse, de repli sur soi, de perte d'énergie, d'apathie, de fatigue accrue peuvent être considérés comme des symptômes courants du vieillissement (Wasylenki, Martin, Lennox, Perry \& Harrison, 1987). On a identifié de nombreux prédicteurs de la dépression chez les personnes âgées: modifications physiologiques survenant avec l'âge; deuils multiples; maladie chronique; diminution du statut social, des ressources socioéconomiques et de la maîtrise de la situation (Clark \& Vorst, 1994; Badger, 1993). Certains troubles physiques plus communs chez les aînés, et les médicaments utilisés pour les traiter, peuvent aussi causer la dépression (Spar \& LaRue, 1990; McCullough, 1991). Ces facteurs sont responsables, en partie du moins, des diagnostics manqués, erronés ou exagérés pouvant avoir des conséquences mortelles. Dans presque tous les pays industrialisés, c'est chez les hommes âgés de 76 ans et plus qu'on note le taux le plus élevé de suicide (Pearson et Conwell, 1995). Le traitement de la dépression peut prendre plusieurs formes. Lorsqu'un diagnostic de dépression clinique est posé, il peut nécessiter diverses interventions (par ex., soutien émotionnel, renforcement du système de soutien, psy- chothérapie, administration de médicaments, traitement des troubles physiques et traitement de choc) faisant appel à de nombreuses disciplines. Mossey, Knott, Higgins et Talerico (1996) ont découvert qu'il existait un lien entre la dépression des personnes âgées et le déclin physique et la perte de statut social, et que les interventions psychosociales étaient des stratégies de traitement efficaces dans certains cas. Il est essentiel d'affecter davantage de ressources à la prévention, au dépistage et au traitement précoces de la dépression, ainsi qu'à la réadaptation psychosociale.

Les troubles délirants ou psychoses des personnes âgées (qui se traduisent par des idées délirantes, des hallucinations, des troubles de la pensée, un comportement bizarre, ou toute autre preuve de perte de contact avec la réalité) peuvent constituer un trouble à part entière ou la manifestation d'autres troubles, tels que la démence, le délire ou la dépression (Jeste, Harris, \& Paulsen, 1996). Déterminer, et gérer, les causes sous-jacentes et les facteurs contributifs des troubles délirants de la vieillesse, qu'ils soient physiques ou sociaux, est une tâche complexe qui nécessite l'intervention de plusieurs disciplines. Les délires de vol ou de jalousie sont souvent associés à la démence et source de beaucoup de stress pour les soignants familiaux (Rockwell, Jackson, \& Vilke, 1994). Le délire peut s'accompagner d'hallucinations ou d'idées délirantes, et il arrive qu'en traitant avec succès les symptômes psychiatriques, on néglige la véritable cause du délire. Les idées délirantes classiques, qui peuvent être la continuation d'une psychose antérieure ou l'apparition d'une psychose récente, nécessitent le même traitement que celles des autres adultes, à ceci près que le dosage médicamenteux doit être adapté. Certaines personnes souffrant de troubles délirants peuvent refuser de se faire traiter, ou leur traitement peut s'avérer inefficace (Tuokko, Frerichs, Halpern, \& Eisner, 1999). Lorsque tel est le cas, il faut travailler en priorité avec le réseau de soutien social du client pour l'amener à mieux comprendre, et accepter, le comportement aberrant, et stabiliser ce système de soutien pour maintenir un milieu aussi familier que possible pour le patient.

\section{Obstacles à l'accès aux soins}

Les obstacles à l'accès des personnes âgées aux services communautaires de santé mentale offerts traditionnellement aux adultes viennent à la fois du système et des clients (Zarit, 1980; German, Shapiro, \& Skinner, 1985). L'attitude négative de la société à l'égard des aînés se reflète chez de nombreux spécialistes de la santé mentale (Paterson, 1976; Butler, 1975). Le problème de l'accès aux services est particulièrement préoccupant pour les personnes âgées qui ne sont pas toujours capables de quitter physiquement 
leur domicile, ou l'établissement de soins de longue durée qui est devenu leur domicile (Knight, 1994). On a aussi lié la sous-utilisation des centres communautaires de santé mentale par les personnes âgées au stigmate de la «maladie mentale», les aînés associant, en effet, les troubles mentaux à un échec personnel ou un manque de foi (Lebowitz, 1988). Au Canada, on a pris des mesures pour éliminer au moins quelquesuns de ces obstacles en mettant en place des programmes communautaires de psychogériatrie (Stolee, LeClair, \& Kessler, 1994; Wargon \& Shulman, 1987).

La plupart des personnes âgées atteintes de troubles mentaux peuvent être traitées chez elles, avec de l'aide, ou en établissement de soins. Toutefois, un grand nombre d'aînés ont souvent d'autres problèmes qui rendent plus difficile la satisfaction de leurs besoins. Ainsi, à mesure que la population des malades mentaux chroniques (tels que maniacodépressifs, schizophrènes) augmentera, certains souffriront de complications physiques ou psychiatriques dues à l'âge. Comme les malades mentaux ont une espérance de vie plus longue, il y a fort à parier qu'ils auront besoin de services à domicile ou en établissement à l'avenir, cela est particulièrement vrai pour les personnes atteintes du syndrome de Down qui ont davantage de risque de développer des démences de type maladie d'Alzheimer (Janicki \& Dalton, 1999). Certaines personnes souffrant de démence, ou d'autres troubles psychiatriques, ont des crises d'agressivité imprévisibles qui ne peuvent pas toujours être contrôlées et posent de sérieux problèmes sur le plan de la prise en charge. Un trouble de la personnalité, par exemple, peut être dissimulé par un ou plusieurs facteurs aggravants: détérioration de l'état physique; crise sociale ou médicale; alcoolisme; comportement social inadapté; comportement antisocial ou psychotique; agressivité physique. Les aînés issus de groupes minoritaires qui ont des besoins culturels et linguistiques particuliers et souffrent aussi de troubles mentaux peuvent avoir de la difficulté à s'intégrer au système traditionnel de soins aux personnes âgées.

Chacune des populations dont nous venons de parler a des besoins spéciaux auxquels ne répondent pas forcément les services conventionnels destinés aux personnes âgées (c.-à-d., établissements de soins de longue durée ou soutien à domicile), ni les services de santé mentale pour adultes. Pour prendre en charge ces populations, il faudra former les professionnels aux questions de santé mentale dans une grande variété de milieux de soins (tels que services médicaux, établissements de soins de longue durée, services de lutte contre l'alcoolisme et la toxicomanie, programmes multiculturels). Certains de ces fournisseurs de soins ne connaissent pas toujours les besoins de la population psychogériatrique ou craignent de s'occuper d'une personne atteinte de trouble mental. Par ailleurs, ceux qui travaillent déjà avec cette population devront être formés aux besoins particuliers de certains groupes. L'évaluation et la prestation des soins nécessiteront la collaboration des divers services. Pour ce faire, il faudra peut-être transférer la gestion des cas d'un service à l'autre en fonction des besoins et mettre en place, ou élargir, des approches et ressources spécialisées (par ex., programmes de jour, unités de soins spéciaux, unités d'évaluation et de traitement psychogériatrique en établissement, établissements de soins tertiaires) pour permettre à ces populations de s'intégrer au système traditionnel de soins aux personnes âgées. Il faudra aussi adopter des stratégies afin d'uniformiser les pratiques des organismes (par ex., signalement de la violence et de la négligence à l'égard des personnes âgées, clients communs) et trouver des façons d'offrir les services les plus appropriés.

\section{Facteurs de développement et facteurs sociaux}

Il est important que ceux qui travaillent avec des personnes âgées reconnaissent les signes du vieillissement normal et ne les confondent pas avec des réactions pathologiques. Les maladies chroniques et affections dégénératives sont plus fréquents chez les personnes âgées que chez les autres adultes (Statistique Canada, 1990) et peuvent se traduire par toutes sortes de symptômes (tels que malaises, diminution de la résistance, de la vigueur et de la mobilité) qui nécessiteront des mesures correctives.

Comme ils sont davantage sujets aux maladies, les aînés prennent plus de médicaments que les autres adultes. Les changements physiologiques liés au vieillissement affectent la résorption, la distribution, la désintoxication et l'élimination des médicaments (Martin, 1987) et se traduisent par une vulnérabilité accrue aux effets secondaires. Ils peuvent entraîner une perte d'autonomie qui limitera les personnes âgées dans l'exercice de leurs activités quotidiennes, nuira à leur vie sociale ou les empêchera de rester chez elles. En supprimant ou en réduisant les médicaments ayant des effets préjudiciables, on peut obtenir de nettes améliorations. Les fournisseurs de services de santé qui travaillent avec cette clientèle doivent donc bien connaître les médicaments le plus souvent prescrits, et les effets idiosyncratiques que ces médicaments, pris seuls ou avec d'autres, peuvent avoir sur la santé.

Les deuils, l'isolement social et la solitude peuvent prédisposer les personnes âgées aux troubles mentaux. On pense ici à la perte d'un conjoint, d'amis et de frères et sœurs à la suite d'un décès, d'une maladie invalidante, d'un handicap physique et/ou d'un dys- 
fonctionnement cognitif. Il sera peut-être possible d'empêcher l'apparition des troubles mentaux en aidant les personnes âgées à faire face à ces deuils et à combler les «vides». Il est important que ceux qui travaillent avec les personnes âgées les aident à surmonter ces pertes qui mettent en péril leur santé mentale, à renouer avec leur cercle d'amis ou à lier de nouvelles relations sociales, il faut aussi qu'ils comprennent en quoi consistent les deuils sociaux liés à l'âge et comment leurs effets cumulatifs agissent sur le moral des aînés.

\section{De la théorie à la théorie}

Les Lignes directrices (Santé et Bien-être social Canada, 1988a) et la définition élargie de la santé mentale, permettant de planifier les services et de répondre aux besoins en santé mentale des personnes âgées, ont été adoptées alors qu'on s'efforçait de redéfinir le système de santé et de passer des soins en établissement aux soins en milieu communautaire. Cette convergence d'esprit aurait pu nous faire croire que nous étions à l'aube d'un nouvel ordre d'où émanerait un système modèle capable de répondre aux besoins en santé mentale des personnes âgées.

Le système de santé actuel n'a toujours pas atteint les objectifs décrits dans les Lignes directrices et ce, pour plusieurs raisons. En voici trois qui expliquent très bien cet échec:

1. on continue de se fier au diagnostic médical pour mesurer les résultats;

2. le coût des services de santé demeure la préoccupation dominante;

3. les services communautaires traditionnels n'ont pas été conçus en pensant aux personnes âgées atteintes de troubles mentaux, et les besoins de cette population ne peuvent pas toujours être satisfaits par les services offerts.

\section{L'accent sur la médecine}

En continuant à privilégier le diagnostic médical pour mesurer les résultats des programmes de santé mentale, on restreint la gamme des services dispensés, mais aussi la structure, la façon de penser et les stratégies de résolution des problèmes de l'équipe de soignants. Selon McCubbin (1994), les modèles d'intervention professionnelle (rôle de l'équipe et gestion des cas) sont devenus des structures hiérarchiques contrôlées, ou conçues, par les psychiatres. Le pouvoir de la psychiatrie repose sur un système qui, nous l'avons $\mathrm{vu}$, évalue le bien-fondé des programmes de santé mentale en fonction du nombre de cas diagnostiqués. Selon Kenny (1996), l'équipe multidisciplinaire en tant que groupe égalitaire où l'avis de chacun est pris en compte serait un mythe. Elle souligne que le langage utilisé pour discuter des cas et monter les dossiers des patients est largement influencé par la perspective médicale, les autres disciplines adaptant leur propre terminologie et perspectives pour être plus crédibles. Le point de vue des disciplines non médicales est donc étouffé, au détriment des personnes âgées atteintes de troubles mentaux et de leurs familles.

Les équipes, en tant que petits groupes, sont soumises à la dynamique du groupe, laquelle influe, à son tour, sur le fonctionnement de l'équipe. Des études (Butterill, O'Hanlon \& Book, 1992) ont établi l'importance de la dynamique de groupe sur la constitution d'équipes fonctionnelles cohérentes, mais, en pratique, les programmes n'ont pas de temps à consacrer à monter de telles équipes (MacCourt, 1993). Le modèle qui semble dominer est donc un modèle hiérarchique où les médecins sont au sommet de la pyramide, ce qui va à l'encontre des valeurs, objectifs et $\mathrm{du}$ fonctionnement du modèle social de soins convenant le mieux aux personnes âgées atteintes de troubles mentaux (Knight \& Kaskie, 1995; Santé et Bien-être social Canada, 1988a; Blazer \& Maddox, 1982).

La prémisse sur laquelle repose l'efficacité de ce modèle appliqué aux services de santé est que les connaissances, les compétences et l'aptitude du groupe à résoudre les problèmes sont plus importants, collectivement, que la somme des parties. Cette prémisse n'est valable que si chaque membre de l'équipe bénéficie du même respect et de la même considération que les autres, et que cela se reflète dans les actions, les relations et la répartition du pouvoir entre les membres de l'équipe. Le bon fonctionnement de l'équipe doit être clairement encouragé, car on sait qu'une équipe dysfonctionnelle peut empêcher les membres d'atteindre leurs objectifs communs. Il est donc important de promouvoir la constitution d'équipes multidisciplinaires homogènes. Pour ce faire, il faut assurer le maintien de la formation et des ressources des équipes, et trouver des façons d'identifier et de régler les problèmes des équipes dysfonctionnelles lorsqu'ils se présentent.

En plus d'influer sur le bon fonctionnement de l'équipe, le fait de privilégier le diagnostic des psychiatres pour mesurer les résultats des programmes, limite les interventions non médicales ou non pharmaceutiques, dont l'efficacité est pourtant attestée. Mittelman et al. (1993) ont démontré qu'un programme complet de counseling pour les conjoints - soignants pouvait retarder le placement en établissement des personnes atteintes de la maladie d'Alzheimer. Carstensen et Fremouw (1981) ont prouvé qu'une approche comportementale clinique permettait de gérer efficacement la paranoïa chez les vieil- 
lards. Macaskill (1987) signale un cas de parasitose délirante traitée avec succès par des moyens non pharmacologiques. Selon Chadwick et Lowe (1994), la thérapie cognitive permet de modifier les idées délirantes. On a établi que la psychothérapie pouvait être aussi efficace que les médicaments pour traiter, en consultation externe, les personnes âgées souffrant de dépression. Ainsi, dans une étude quantitative sur la psychothérapie, Scogin et McElreath (1994) relèvent 17 cas où la psychothérapie, en l'absence de tout autre traitement, a donné des résultats positifs, et concluent que les résultats enregistrés sont semblables à ceux d'études comparant l'effet d'un traitement médicamenteux à l'effet placebo. En Australie, Rosewarne, Bruce et McKenna (1997) ont démontré que la consultation et l'élaboration de programmes en établissement peuvent être bénéfiques pour la santé mentale des clients et permettre aux foyers de s'occuper plus longtemps des pensionnaires atteints de démence.

Bien que les chercheurs aient démontré l'utilité des mesures de prévention de la dépression chez les aînés (par ex. Gallagher et al., 1989; Murphy, 1982; Turner \& Noh, 1988), l'enquête effectuée par MacCourt (1993) révèle que les services de santé mentale pour personnes âgées de l'Ouest du Canada font très peu de travail préventif. Selon cette enquête, menée auprès de 28 programmes communautaires de psychogériatrie de l'Ouest du Canada, le personnel consacre environ 80 pour cent de son temps à la prestation de services directs aux clients, et 20 pour cent seulement à l'éducation de l'entourage, la recherche, le développement et la consultation communautaires, bien que cela fasse clairement partie de son mandat (MacCourt, 1993). Le temps passé à la promotion de la santé, la consultation, l'éducation et le développement communautaire, qui pourraient permettre le dépistage ou la prévention précoces des troubles mentaux et leur gestion, était négligeable. Comme les autres activités (telles que travail de prévention auprès des personnes atteintes de troubles psychiatriques plutôt que de maladie mentale, ou sensibilisation des établissements) ne «comptent» pas, il n'est pas surprenant que les programmes concentrent leurs efforts sur les malades mentaux les plus gravement atteints afin de «s'en débarrasser» rapidement. Ces résultats vont à l'encontre de ce que préconisent les Lignes directrices (Santé et Bien-être social Canada, 1988a), à savoir que la promotion de la santé, la prévention et l'intervention précoces sont des fonctions importantes des programmes. Il est capital que ces activités soient reconnues comme des activités légitimes des programmes de psychogériatrie.

\section{Réduire le coût des services de santé}

La rationalité technique est le deuxième grand facteur influant sur la prestation des services de santé men- tale aux personnes âgées. Ce concept relativement nouveau, mais très important, sert à définir les besoins des aînés atteints de troubles mentaux, et à déterminer qui devrait dispenser les services et comment les dispenser. La rationalité technique est décrite ainsi:

pratique épistémologique dérivée de la philosophie positiviste... selon laquelle les praticiens sont des résolveurs de problèmes instrumentaux qui choisissent les moyens techniques les mieux adaptés pour atteindre certaines fins. Ces praticiens rigoureux résolvent des problèmes bien formés et instrumentaux en appliquant les théories et techniques tirées de connaissances systématiques, de préférence scientifiques (Schon, 1987, p.4).(Traduction libre)

La domination continue du modèle médical, mentionnée plus tôt, est renforcée par l'importance que l'on accorde actuellement à la technocratie dans les services de santé. Ces perspectives (médicale et technocrate) sont aussi respectées que la «science» et le «fait empirique» et accordent plus de poids à «l'objectivité» et aux activités «dures» qu'aux «relations», à la «compassion» et aux activités «douces». Chappell (1994) suggère que les changements notés dans la prestation des soins médicaux et dans les hôpitaux viennent de ce que les médecins relèvent de cette nouvelle classe d'administrateurs.

La rationalité technique est censée être neutre et objective, mais il est permis d'en douter. Moody (1987) rejette l'idée même de pragmatisme objectif qu'il décrit comme étant:

un jugement de valeur se cachant derrière des critères techniques ( $p$. ex., échelles de mesure pour déterminer l'admission dans un établissement) ou autres et nous permet de dissimuler nos valeurs derrière un jargon professionnel masquant la réalité du choix humain et la responsabilité qu'il suppose. (p. 13). (Traduction libre)

L'objectif de la rationalité technique est évidemment de réduire le coût des services de santé. Les décisions prises pour atteindre cet objectif s'appuient sur des valeurs qui sont étrangères au concept de soins, mais qui, pourtant, déterminent le type de soins à dispenser et la façon de les dispenser. Lorsque le seul critère de sélection des services offerts aux personnes âgées atteintes de troubles mentaux est le coût, on aurait tort de s'attendre à ce que les besoins de la clientèle psychogériatrique soient satisfaits. Le choix des services importants ou nécessaires repose sur des valeurs et, dans le climat actuel, c'est la valeur monétaire qui domine les décisions en matière de politiques.

Bien que les politiques de santé actuelles visent à réduire l'utilisation des lits de soins actifs et de longue 
durée, (et donc à minimiser le coût des services de santé), les services communautaires n'ont, pour ainsi dire, reçu aucun fonds pour faciliter ce transfert (Chappell, 1993). On sait que de bons soins communautaires coûtent au moins aussi cher que des soins en établissement (Lamb, 1993). Il semble que les coupures budgétaires dans le système de santé aient entraîné une diminution des services en établissement et en milieu communautaire, et créé des problèmes encore plus profonds. Les familles doivent désormais combler les lacunes dues aux pressions exercées sur le système de santé, qu'elles aient ou non les ressources financières ou humaines nécessaires (Walker, 1991). De nombreuses études ont été faites sur le fardeau des soignants, et l'on sait que s'occuper d'une personne atteinte de démence est beaucoup plus stressant que s'occuper de quelqu'un qui souffre d'un trouble physique (Chappell, 1990). À l'heure actuelle, les familles de soignants, n'étant pas les clients désignés, reçoivent très peu de soutien (p. ex., émotionnel ou instrumental; sensibilisation).

Selon Havens (1995), il est avéré qu'on ne peut faire d'économies dans le secteur de la santé en réduisant le personnel des programmes communautaires de soins de longue durée. Elle fait remarquer qu'en imposant une charge de travail excessive au personnel on l'empêche d'évaluer rapidement les cas, d'examiner les dossiers en attente, de procéder aux réévaluations en temps utile et de trouver des solutions originales. De ce fait, certains clients reçoivent davantage de services qu'ils n'en ont besoin et d'autres moins, ces deux extrêmes étant des conséquences coûteuses de la mauvaise dotation en personnel des programmes (Havens, 1995).

Dans le contexte actuel de réforme de l'ensemble du système de santé, l'accent est mis sur la mesure des résultats pour déterminer l'efficacité des services au lieu de se fier uniquement à l'analyse des coûts (Hargreaves, Shunway, Hu, \& Cuttel, 1998; Lyons, Hoawrd, O'Mahoney, \& Lish, 1997). Pourtant, il est intéressant de noter que, face à cette tâche, nombre de fournisseurs de services de santé mentale sont incapables d'énoncer leurs objectifs en matière de programmes et de clients, sauf dans les termes les plus vagues (comme ceux des Lignes directrices fédérales). Il faut concevoir des mécanismes d'évaluation qui privilégient les objectifs (par ex., objectifs du programme/du système: limiter la polypharmacie, limiter les hospitalisations et/ou les placements en établissement inutiles; objectifs en matière de clients: soulager les symptômes, augmenter l'autonomie, améliorer la qualité de vie, limiter le stress des soignants) et ne se contentent pas de comptabiliser le nombre de clients évalués. En mettant l'accent sur les objectifs on favorisera le recours à des «pratiques cli- niques exemplaires» qui énoncent clairement les besoins, encouragent l'innovation et permettent aux fournisseurs de services de santé mentale de prouver qu'ils ont un rôle important à jouer au sein du système de santé et dans la vie de leurs patients.

Malgré les besoins, peu de cliniciens ont les connaissances ou le temps de préparer et d'évaluer les programmes. Les évaluations de résultats mal conçues ou mal faites par des cliniciens ou bailleurs de fonds peuvent être très préjudiciables aux programmes. Il serait donc utile d'obtenir la collaboration des chercheurs de la santé pour pallier cette lacune.

Limites du système de santé communautaire officiel

La plupart des services communautaires auxquels ont recours les personnes âgées ne s'adressent pas aux clients de psychogériatrie et sont souvent inadéquats. Comme les services sont limités, les clients de psychogériatrie se trouvent en concurrence avec ceux pour qui ces services ont été conçus au départ (par ex., les personnes handicapées n'ayant pas de troubles mentaux). En Colombie Britannique, les programmes de santé mentale destinés aux personnes âgées ont peu de services qui leur soient propres, ils ont accès au soutien à domicile et aux soins en établissement par le biais des programmes de soins aux malades chroniques ou de soins de longue durée. Les services de santé mentale (tels que foyers de groupes pour malades mentaux, emplois réservés, services de loisirs et de réadaptation) qui sont offerts aux autres adultes atteints de troubles mentaux sont rarement accessibles aux aînés de plus de 65 ans. Les soins de relève et les soins de longue durée, destinés aux personnes âgées fragiles ayant des problèmes de santé chroniques, desservent une population plus frêle, malade et âgée qu'il y a dix ans (Havens, 1995). Un nombre croissant de personnes âgées atteintes de démence continuent de vivre dans la communauté, souvent seules et dépendantes des services communautaires (Tuokko, MacCourt, \& Heath, 1999; Webber, Fox, \& Burnette, 1994).

Avec l'apparition de nombreux troubles mentaux à la vieillesse, il devient urgent de régler les questions de logement et de soins en établissement. Les établissements de soins de longue durée sont mal équipés pour accueillir une clientèle chez qui la déficience intellectuelle est de plus en plus courante et qui a besoin d'une structure, de repères environnementaux et sociaux et de prévisibilité pour fonctionner le mieux possible (Milke, Dobbe, Rule, \& Milke, 1987). Dans une étude menée en C.-B. sur les problèmes que pose la prestation de soins en établissement aux clients de psychogériatrie, Gutman, MacFadgen et Killam (1995) indiquent que les niveaux actuels de dotation en personnel de première ligne sont insuff- 
isants et que le personnel n'a pas la formation nécessaire pour gérer les troubles mentaux et comportementaux des patients. La création des unités de soins spéciaux (USS), où l'on traite l'errance ou autres problèmes de comportement des personnes âgées dus à une démence moyenne à grave, semble indiquer qu'il est possible d'adapter les programmes et d'améliorer la qualité des soins.

À l'heure actuelle, certains clients des services de santé mentale ne peuvent être admis en établissement de soins si leur comportement est «agressif» ou «difficile», ou si l'on juge qu'ils risquent d'être difficiles à gérer pour le personnel ou à intégrer dans l'établissement (Gutman et al., 1995). Les lits de soins tertiaires sont fermés ou ne sont pas disponibles. Ces clients doivent donc rester chez eux plus longtemps qu'ils ne le devraient en attendant qu'un lit se libère dans un service approprié, ou attendre d'être placés dans un service de soins actifs dont l'exploitation coûte cher. Il est donc impératif $\mathrm{d}$ 'offrir la formation et le soutien requis au personnel de première ligne des établissements et des services communautaires pour lui permettre de relever les défis qu'il ne manquera pas de rencontrer. Notre priorité doit être d'avoir assez de personnel convenablement formé et de milieux de soins, adaptés ou nouveaux, pour répondre aux besoins particuliers de cette population difficile.

\section{Un système de services complets}

On trouvera, dans les Lignes directrices pour la prestation de services complets aux personnes âgées atteintes de troubles mentaux (Santé et Bien-être social Canada, 1988a), la description détaillée de certains principes directeurs régissant la planification des programmes et services destinés aux aînés canadiens atteints de troubles mentaux. Voici quelques points clés:

- il faut s'assurer de la participation d'une grande variété de professionnels, ressources communautaires et personnel de soutien;

- les services d'action communautaire sont essentiels et doivent comporter l'évaluation des patients à domicile et la consultation des établissements de soins de longue durée;

- l'évaluation, le traitement et la planification reposent sur un cadre général et des équipes multidisciplinaires;

- on met l'accent sur la continuité et la coordination des soins.

Aucune discipline n'a toutes les connaissances nécessaires pour comprendre et traiter les besoins en services de santé mentale des personnes âgées qui, nous l'avons $\mathrm{vu}$, peuvent être d'origine physiologique, pharmacologique, psychologique et sociale. Il faut faire appel aux connaissances et à l'expertise de plusieurs disciplines - médecins de famille, spécialistes, tels que psychiatres et gériatres, ergothérapeutes, personnel infirmier, travailleurs sociaux et psychologues - chacun ayant des connaissances spécialisées sur les personnes âgées et le vieillissement selon leur discipline. Les équipes multidisciplinaires utilisant diverses approches biologiques, sociales, psychologiques et environnementales et capables d'offrir des services communautaires, des évaluations et traitements à domicile, et de la formation aux soignants professionnels et bénévoles, sont désormais considérées comme essentielles pour répondre aux besoins en services de santé mentale des aînés dans la communauté et dans les établissements de soins de longue durée (Wargon \& Shulman, 1987; Jones, 1991). Il est évident que seuls les grands centres pourront offrir ce modèle de pratique idéal et, même dans ce cas, il faudra qu'un membre de l'équipe réunisse les renseignements initiaux en contactant ou consultant les membres des autres disciplines, selon le besoin. Dans les petits centres où il n'est pas toujours possible de consulter directement les spécialistes d'autres disciplines, on pourra organiser des téléconférences ou des vidéoconférences.

Voici quelques-uns des objectifs généraux visés par la politique de services de santé mentale aux personnes âgées (Santé et Bien-être social Canada, 1988a):

- $\quad$ réduire la détresse des patients et de leurs familles;

- améliorer et maintenir la capacité fonctionnelle;

- aider la personne à mobiliser son énergie pour vivre de façon autonome;

- $\quad$ optimiser et maintenir l'autonomie au plus haut niveau possible.

Les rôles et fonctions essentiels des services spécialisés de psychogériatrie ont été décrits (Santé et Bienêtre social Canada, 1988a) comme suit:

- $\quad$ diagnostic des cas complexes, ensuite confiés au médecin de famille pour traitement;

- $\quad$ élaboration de plans de traitement pouvant nécessiter des séances de psychothérapie pour le patient ou sa famille, l'apprentissage de techniques de modification du comportement, ou une combinaison de services spéciaux, tels que physiothérapies, traitements de choc, psychothérapie et action communautaire, coordination des services;

- évaluation touchant les questions de droit, p. ex., déterminer la capacité mentale, conseiller les familles sur les conséquences de cette détermination et les aider à démêler les complexités juridiques qui en résultent;

- $\quad$ consultation avec les organismes et programmes communautaires, services de gériatrie et services médico-chirurgicaux d’hôpitaux généraux et 
d'établissements de soins de longue durée: ce type de consultation vise à modifier le milieu social, notamment l'attitude du personnel ou la dynamique des relations familiales;

- $\quad$ promotion de la recherche, de l'éducation et de la formation sur le terrain, y compris sensibilisation du public aux besoins et à la prise en charge des personnes âgées atteintes de troubles mentaux.

L'un des principaux objectifs de la psychogériatrie est de permettre à la personne de rester chez elle, pour ce faire il faudra peut-être faire appel à une variété de services: publics, privés et bénévoles.

L'examen des principes énoncés dans les Lignes directrices (Santé et Bien-être social Canada, 1988a) semble indiquer que les services de santé mentale destinés aux personnes âgées s'inspirent d'un modèle social de prestation des soins et font appel aux perspectives, aux connaissances et à l'expertise de professionnels de la santé de plusieurs disciplines. Le modèle social nous fournit le cadre nécessaire à l'étude de l'ensemble des facteurs internes et externes contribuant aux troubles mentaux, et des interventions qui peuvent être médicales, psychologiques, sociales ou environnementales (Novak, 1988). En général, les modèles sociaux de prestation des soins sont axés sur les clients et les plans de soins privilégient les besoins des clients et de leur réseau de soutien social bénévole (Havens, 1995). On sait aussi qu'il faut parfois chercher l'origine d'un trouble mental dans des circonstances indépendantes de la personne (par ex., dépression due à l'isolement social qui, à son tour, est le fait de la pauvreté). En Amérique du Nord, les soignants familiaux accomplissent entre 70 et 80 pour cent des soins de santé, soins personnels et aide aux tâches essentielles requises par les personnes âgées (Nahmiash, 1995). Pourtant, la présence d'un aîné ayant besoin de soins est une source courante de stress dans les familles et peut être particulièrement difficile pour le soignant principal. L'anxiété, la culpabilité, la fatigue, la maladie physique, les contacts sociaux limités et la dépression sont souvent le lot de l'adulte qui s'occupe d'un parent âgé (Clark, 1987). C'est pourquoi évaluer les besoins des soignants et la suffisance des soutiens sociaux, les aider à démêler les sentiments complexes liés à la prise en charge et leur offrir un soutien affectif qui leur permette d'équilibrer leurs besoins et ceux de la personne dont ils s'occupent sont des activités importantes qui nécessitent les compétences de plusieurs disciplines. La prestation de soins appropriés consiste en une vaste gamme d'interventions indirectes, telles que consultation avec d'autres professionnels et organismes; participation aux efforts communautaires pour créer ou améliorer les services de soutien offerts; travail préventif (par ex., groupes s'occupant de clients sujets à la dépres- sion); défenses des droits des patients et sensibilisation de l'entourage. Pour fonctionner, ce modèle doit s'appuyer sur une équipe multidisciplinaire et non sur les membres de disciplines individuelles travaillant chacun de leur côté.

Le modèle social de prestation des soins repose sur une conception élargie et systémique des troubles mentaux qui dépasse le diagnostic médical et englobe l'évaluation et la gestion des conséquences de la maladie pour le réseau social de la personne, ses relations humaines et son environnement et vice versa. Les médecins ou psychiatres devront peut-être participer au traitement des troubles médicaux comorbides ou à la symptomatologie psychiatrique, mais le diagnostic et le traitement de la maladie mentale n'est qu'une des facettes des soins de la personne. Il est donc clair que pour traiter des troubles mentaux complexes toute discipline agissant seule est forcément limitée.

\section{Résumé}

Bien que le gouvernement fédéral ait adopté un définition élargie de la santé mentale et des lignes directrices visant à fournir des services psychogériatriques à portée sociale (Santé et Bien-être social Canada, 1988a,b), il s'est avéré difficile de mettre en œuvre ces idéaux dans le cadre actuel des services de santé. On a vu que les personnes âgées atteintes de troubles mentaux ont de nombreux besoins qui ne sont pas médicaux, pourtant le financement de leur prise en charge en milieu communautaire et dans les établissements de soins reste axé sur les soins de santé. Les fonds viennent presque exclusivement du budget de la santé où les services médicaux sont rigoureusement comptabilisés. Il faudrait adopter la perspective mieux équilibrée décrite dans les Lignes directrices (Santé et Bien-être social Canada, 1988a). La gamme actuelle de services communautaires est inadéquate et insuffisante pour répondre aux besoins des personnes âgées atteintes de troubles mentaux, pourtant, il semble qu'on n'ait prévu d'allouer aucun fonds, dans l'immédiat, à l'élargissement ou l'amélioration de ces services. Comme l'indiquait le Conseil consultatif national sur le troisième âge «Pour réformer le système de santé et le rendre abordable, il ne suffira pas de contrôler les coûts, il faudra dispenser des soins appropriés et efficaces adaptés aux besoins changeants des Canadiens.» (Traduction libre) (CNTA, 1995b, 20). Les besoins en services de santé mentale des aînés canadiens doivent être étudiés avec soin. Il est à souhaiter que la volonté actuelle de réforme du système de santé apportera l'élan nécessaire pour transformer radicalement les modèles de soins s'adressant à ce groupe extrêmement vulnérable de personnes âgées. 


\section{Références}

Association médicale canadienne (1987). Health care for the elderly: today's challenges: tomorrow's options. Ottawa, ON: Association médicale canadienne.

Badger, T. (1993). Physical health impairment and depression among older adults. IMAGE: Journal of Nursing Scholarship, 25(4), 325-330.

Béland, F. (1997). Editorial: Building Canadian gerontology: a springboard for international recognition? Revue canadienne du vieillissement, 16(1), 6-10.

Beresin, E. (1988). Delirium in the elderly. Journal of Geriatric Psychiatry and Neurology, 1(3), 127-143.

Blazer, D., \& Maddox, G. (1982). Using epidemiologic survey data to plan geriatric mental health services. Hospital and Community Psychiatry, 33(1), 42-45.

British Columbia Psychogeriatric Association (1999). Principles of Psychogeriatric Care. (Available from the British Columbia Psychogeriatric Association, 528 Wentworth Street, Nanaimo, BC, V9R 3E4.)

Butler, R.N. (1975). Psychiatry and the elderly: an overview. American Journal of Psychiatry, 132(9), 893-900.

Butterill, D., O'Hanlon, J., Book, H. (1992). When the system is the problem, don't blame the patient: Problems inherent in the interdisciplinary inpatient team. Revue canadienne de psychiatrie, 37 (Avril), 168-172.

Carstensen, L., \& Fremouw, W. (1981). The demonstration of a behavioural intervention for late life paranoia. Gerontologist, 21(3), 329-333.

Chadwick, P. \& Lowe, C. (1994). A cognitive approach to measuring and modifying delusions. Behav. Re. Ther., 32(3):355-367.

Chappell, N. (1990). Aging and social care. Handbook of Aging and the Social Sciences, Third edition, pp. 438-454. Academic Press, Inc.

Chappell, N. (1993). The future of health care in Canada. Journal of Social Policy, 22(4), 487-505.

Chappell, N. (1994). Home care research: what does it tell us? The Gerontologist, 34(1), 116-120.

Clark, D. (1987). In Wasylenki et al. (Eds.), Psychogeriatrics: a practical handbook, Toronto: Gage Educational Publishing Co.

Clark, W., \& Vorst, V. (1994). Group therapy with chronically depressed geriatric patients. Journal of Psychosocial Nursing, 32(5), 9-13.

Conseil consultatif national sur le troisième âge (CNTA) (1995). The NACA position on community services in health care for seniors: Progress and challenges. Ottawa: CNTA.

Gallagher, D., Rose, J., Rivera, P., et al. (1989). Prevalence of depression in family caregivers. Gerontologist, 29(4), 449-456.

German, P., Shapiro, B. \& Skinner, E. (1985). Mental health of the elderly: use of health and mental health services. Journal of the American Geriatrics Society, 33(4), 246-252.

Groupe d'étude sur la santé et le vieillissement au Canada. (1994). Canadian study of health and aging: study methods and the prevalence of dementia. Journal de l'Association médicale canadienne,150(6), 899-12.
Gurland, B.J., \& Cross, P.S. (1982). Epidemiology of psychopathology in old age: some implications of clinical services. Psychiatric Clinics of North America, 5(1), 11-26.

Gutman, G., MacFadgen, L., Killam, J. (1995). Psychogeriatric client identification project. Simon Fraser University.

Hargreaves, W. A., Shumway, M., Hu, T., \& Cuffel, B. (1998). Cost-outcome methods for mental health. San Diego: Academic Press.

Havens, B. (1995). Long-term care diversity within the care continuum. Revue canadienne du vieillissement, 14(2), 245-263.

Janicki, M. \& Dalton, A. J. (1999). Dementia, aging and intellectual abilities: A handbook. Philadelphia: Brunner/Mazel, Inc.

Jeste, D., Harris, M., \& Paulsen, J. (1996). Psychoses. In Sadavoy, J., Lazarus, L, Jarvik, L., \& Grossberg, G. (Eds.), Comprehensive review of geriatric psychiatry - II. (pp. 593614). Washington, DC: American Psychiatric Press.

Jones, R. (1991). Community service in old age psychiatry. Current Opinion in Psychiatry, 4, 603-607.

Kenny, N. (1996). Présenté au congrès de la Société canadienne de bioéthique, à Montréal.

Knight, B. (1994). Home-delivered mental health services: An idea whose time has come? Gerontologist, 34(2), 149.

Knight, B.G. \& Kaskie, B. (1995). Models of mental health services delivery to older adults. In M. Gatz (Ed.), Emerging issues in mental health and aging (pp. 231-255). Washington, DC: American Psychological Association.

Lamb, R. (1993). Lessons learned from deinstitutionalization in the U.S. British Journal of Psychiatry, 162, 587-592.

Lebowitz, B.D. (1988). Correlates of success in community mental health programs for the elderly. Hospital and Community Society, 39(7), 721-722.

Lee, M. (1996). Drugs and the elderly: do you know the risks? American Journal of Nursing, 96(7), 25-31.

Lipowski, Z.J. (1989). Delirium in the elderly patient. New England Journal of Medicine, 320(9), 378-381.

Lyons, J.S., Howard, K.I., O'Mahoney, M.T., \& Lish, J.D. (1997). The measurement and management of clinical outcomes in mental health. New York: John Wiley \& Sons, Inc.

Macaskill, N. (1987). Delusional parasitosis: Successful nonpharmacological treatment of a folie-a-deux. British Journal of Psychiatry, 150, 261-263.

MacCourt, P. (1993). A survey of community psychogeriatric outreach programs in western Canada. Thèse de maîtrise non publiée, Université du Manitoba, Winnipeg (Manitoba).

Martin, B. (1987). In Wasylenki et al. (Eds.), Psychogeriatrics: a practical handbook. Toronto: Gage Educational Publishing Co.

McCubbin, M. (1994). Deinstitutionalization: The illusion of disillusion. The Journal of Mind and Behavior, 15(1,2), 3554.

McCullough, P. (1991). Geriatric depression: atypical presentations, hidden meanings. Geriatrics, 46(10), 72-76. 
Milke, D., Dobbe, A., Rule, B., \& Milke, A. (1987). The activities of Alzheimer patients and non-demented residents of a nursing home. Présenté à l'assemblée annuelle de l'Association canadienne de psychologie, Vancouver, C.-B.

Ministry of Health and Ministry Responsible for Seniors, (1996). Policy framework for seniors with mental health problems (ébauche). Province de C.-B. (septembre)

Mittelman, M., Ferris, S., Steinberg, G., Shulman, E., Mackell, J., Ambinder, A., \& Cohen, J. (1993). An intervention that delays institutionalization of Alzheimer's disease patients: Treatment of spouse-caregivers. Gerontologist, 33(6), 730-740.

Moody, H. (1987). Ethical dilemmas in nursing home placement. Generations. Summer, 16-23.

Mossey, J., Knott, K., Higgins, M., \& Talerico, K. (1996). Effectiveness of a psychosocial intervention, interpersonal counselling for subdysthymic depression in medically ill elderly. Journal of Gerontology, 51A(4), M172M176.

Murphy, E. (1982). Social origins of depression in old age. British Journal of Psychiatry, 141, 135-142.

Nahmiash, D. (1995). Informal caregiving: women's care or work? In Sawyer, E. \& Stephenson, M. (Eds.), Continuing the care: the issues and challenges of long-term care (pp. 247-269). Ottawa, ON: CHA Press

Novak, M. (1988). Aging and Society, Nelson, Canada.

Organisation mondiale de la santé et Association mondiale de psychiatrie (1998).Organization of care in psychiatry of the elderly: a technical consensus statement. Aging and Mental Health, 2, (3): 246-252.

Paterson, R.D. (1976). Services for the aged in community mental health centres. American Journal of Psychiatry, 133(3), 271-273.

Pearson, J., \& Conwell, Y. (1995). Suicide in late life: challenges and opportunities for research. International Psychogeriatrics, 7, 131-135.

Rockwell, E., Jackson, E., \& Vilke, G. (1994). A study of delusions in a large cohort of Alzheimer disease patients. American Journal of Geriatric Psychiatry 2, 157-164.

Rosewarne, R., Bruce, A., \& McKenna, M. (1997). Dementia programme effectiveness in long-term care. International Journal of Geriatric Psychiatry, 12, 173-182.

Santé et Bien-être social Canada. (1988a). Lignes directrices pour la prestation de services complets aux personnes âgées atteintes de troubles mentaux. Ottawa, ON: Approvisionnements et Services Canada.

Santé et Bien-être social Canada. (1988b). Troubles mentaux chez les personnes âgées au Canada. Considérations démographiques et épidémiologiques. Ottawa, ON: Approvisionnements et Services Canada.

Santé et Bien-être social Canada. (1990). Guide pour l'établissement de normes régissant les services dispensés aux personnes âgées atteintes de troubles mentaux dans les établissements de soins prolongés. Ottawa, ON: Approvisionnements et Services Canada.

Santé et Bien-être social Canada. (1991). Troubles mentaux chez les personnes âgées au Canada. Considérations démographiques et épidémiologiques. Ottawa, ON: Approvisionnements et Services Canada.

Schon, D. (1987). Educating the reflective practioner. San Francisco. Jossey-Bass.

Scogin, F., \& McElreath, L. (1994). Efficacy of psychosocial treatments for geriatric depression: A quantitative review. Journal of Consulting and Clinical Psychology, 62(1), 69-73.

Shulman, R. (1991). Regionalization of psychiatric services for the elderly. Revue canadienne de psychiatrie, 36, 3-8.

Spar, J., \& LaRue, A. (1990). Concise guide to geriatric psychiatry. Washington: American Psychiatric Press, Inc.

Statistique Canada. (1990). Enquête sur la santé et les limitations d'activités, points saillants: Personnes ayant une incapacité au Canada (Catalogue 83-603). Ottawa, ON: Approvisionnements et Services Canada.

Stolee, P., LeClair, K., \& Kessler, L. (1994). Geriatric psychiatry consultation in the community. Revue canadienne de psychiatrie, 39(October), S27-S33.

Turner, R., \& Noh, S. (1988). Physical disability and depression. Journal of Health and Social Behaviour, 29, 23-27.

Tuokko, H., Frerichs, R., Halpern, S., \& Eisner, M. (1999). Delusional symptomatology as seen by a community outreach team. Aging and Mental Health, 3(2), 136142.

Tuokko, H., MacCourt, P., \& Heath, Y. (1999). Home alone with dementia. Aging and Mental Health, 3(1), 2127.

Walker, A. (1991). The relationship between the state and the family in the care of older people. La Revue canadienne $d u$ vieillissement, 10(2), 94-112.

Wargon, M., \& Shulman, K.(1987). Community psychiatric services for the elderly: the Sunnybrook experience. Santé mentale au Canada, March, 3-6.

Wasylenki, D., Martin, B., Lennox, E., Perry, L., \& Harrison, M. (1987). Psychogeriatrics: a practical handbook. Toronto: Gage Educational Publishing Co.

Webber, P., Fox, P. \& Burnette, D. (1994). Living alone with Alzheimer's disease: effects on health and social service utilization patterns. The Gerontologist, 34(1), 8-14.

Zarit, S.H. (1980). Aging and mental disorders: Psychological approaches to assessment and treatment. New York Free Press.

Exposé de position préparé par Penny MacCourt, M.S.W., Holly Tuokko, Ph.D., et Mary Tierney, Ph.D., à la demande de l'Association canadienne de gérontologie. Approuvé par le conseil à titre de politique officielle de l'ACG le 28 novembre 2000. 